\title{
CALIDAD Y MEJORA DE LA EDUCACIÓN: MEDIACIÓN EN INSTITUCIONES EDUCATIVAS
}

\author{
Quality and improvement of education: mediation in educational \\ institutions
}

\author{
Silvia Pizarro Elizo \\ e-mail: silviapizarro22@gmail.com \\ Universidad de Extremadura. España
}

\begin{abstract}
RESUMEN
Para conseguir una verdadera calidad y mejora de la educación en las instituciones educativas, es necesario lograr y garantizar entornos educativos pacíficos, en los que esté presente una educación en valores. Espacios libres de violencia, espacios integradores, en los que cada sujeto aprenda a convivir con todos los demás, resolviendo los conflictos que broten, de forma pacífica. Para ello se propone utilizar la mediación como herramienta de paz. Aprender a resolver los conflictos de forma adecuada en los centros educativos es fundamental, para asegurar la calidad y mejora de la educación en las instituciones educativas, para el individuo y para la sociedad en la que éste vive.
\end{abstract}

PALABRAS CLAVE: mediación; centro educativo; educación para la paz; valores sociales y cívicos; convivir.

\begin{abstract}
In order to achieve true quality and improvement of education in educational institutions, it is necessary to achieve and guarantee peaceful educational environments in which values education is present. Spaces free from violence, inclusive spaces, in which each subject learns to coexist with all others, resolving conflicts that arise, peacefully. For this purpose, it is proposed to use mediation as a peace tool. Learning to resolve conflicts adequately in schools is fundamental, to ensure the quality and improvement of education in educational institutions, for the individual and for the society in which he lives.
\end{abstract}

KEY WORDS: mediation; educational center; education for peace; social and civic values; coexist.

Recibido/Received: 06/07/2017

Aprobado/Aproved: 22/12/2017

Cómo referenciar este artículo / How to reference this article:

Pizarro Elizo, S. (2018). Calidad y mejora de la educación: mediación en instituciones educativas. Tendencias Pedagógicas, 31, 207-226. doi: http://dx.doi.org/10.15366/tp2018.31.012 
No hay caminos para la paz, la paz es el camino.

Mahatma Gandhi

\section{INTRODUCCIÓN}

Hablar de calidad y mejora de la educación, indiscutiblemente está relacionado con el logro de entornos educativos pacíficos, con educar en valores, y con la práctica de la mediación, por su carácter preventivo y de resolución de conflictos en las instituciones educativas.

"No cabe un discurso en educación sin que necesariamente, de forma explícita o implícita, estén presentes los valores. Sí así fuese, no sería un acto educativo". (Ortega y Mínguez, 2001, p. 14).

"Por todo ello la educación en valores ha de ser considerada como uno de los aspectos fundamentales del desarrollo educativo de los alumnos". (Esteban, 2003, p.100).

Desde los centros educativos debemos fomentar el aprendizaje y práctica de la educación para la paz, con el fin de conseguir que los sujetos se relacionen de forma pacífica en los centros. De esta forma proporcionamos, también, una educación para la ciudadanía, haciendo posible que los individuos de una sociedad se relacionen y resuelvan asuntos unos con otros de forma no violenta, generando avance, desarrollo, riqueza, bienestar para los ciudadanos.

"La violencia es el último recurso de la incompetencia: Isaac Asimov" (García, 2004, p.191).

"Así, no nos vemos obligados a elegir entre una forma de educación que promueve la rentabilidad y una forma de educación que promueve el civismo. La prosperidad económica requiere las mismas aptitudes necesarias para ser un buen ciudadano". (Nussbaum, 2010, p.30).

El hecho de que tanto en la Constitución Española, como en las principales leyes educativas democráticas (Constitución Española de 1978: Artículo 10; Constitución Española de 1978: Articulo 27.2; Boletín Oficial del Estado, núm. 159, 14 julio de 1985,21016; Boletín Oficial del Estado, núm.238,4 octubre de 1990,2893 y 28931; Boletín Oficial del Estado, núm.278, 20 de noviembre de 1995,33654; Boletín Oficial del Estado, núm.307, 24 de diciembre 2002, 45192; Boletín Oficial del Estado, Núm.106, 4 de mayo de 2006,17158; 15 de marzo de 20007 :11007.Boletín Oficial del Estado Núm.295, 9 de diciembre 2013,97858), se haga referencia a la educación en valores, ya sean valores cívicos, valores democráticos, valores constitucionales, se hable de Educación para la paz y se relacione todo ello con la calidad educativa, independientemente del periodo político en que se hayan sancionado estas leyes, nos hace llegar a la certidumbre sobre la conveniencia de educar en este aspecto, para el individuo y para la sociedad en la que éste vive. Debemos pensar que realmente este tipo de educación es fundamental y por tanto debe hacerse efectiva en las instituciones educativas, no admite tibiezas.

"La dimensión moral es inherente a todo comportamiento humano y, por consiguiente, una educación integral no puede descuidarlo" (Quintana, 1988, p.289). 


\section{EL PAPEL DE LOS CENTROS EDUCATIVOS}

En los centros educativos, las aulas presentan realidades heterogéneas, muy variadas, a veces complejas, también enriquecedoras, que hacen necesario este tipo de educación. Es básico educar en valores al individuo, educar para conseguir entornos inofensivos, de no violencia en sus diferentes manifestaciones, posibilitar que los sujetos aprendan también a solucionar situaciones diversas con otros, de forma conciliadora, prioritariamente desde el ámbito familiar "La familia debe tomar conciencia de sus obligaciones, y saber situarse educativamente ante sus hijos porque no la podemos reemplazar. Sí podemos colaborar, estar cerca, coordinar nuestras acciones y ayudarnos mutuamente en esta estrategia educativa". (Blanchard y Muzas, 2007, p.7). Pero también desde el ámbito educativo, desde las instituciones educativas, es necesario enseñar en la paz, educar en valores, debemos enseñar a resolver controversias, de un modo práctico, funcional en el que los alumnos/as observen, participen, aprendan, ejerciten y apliquen lo aprendido como forma de vida, garantizando entornos educativos pacíficos, supone además un aprendizaje preparatorio, para su posterior incorporación a la vida social activa.

Los colegios e institutos, deben ser dominios en los que los sujetos se relacionen unos con otros de forma positiva, en todas las zonas. Sitios donde no se permita ningún tipo de violencia, ámbitos donde la formación, y el crecimiento personal y social, esté garantizado, lugares seguros, donde en todo momento se respeten los derechos de los individuos, los derechos humanos.

Para Jares, (2004, p.49), los derechos humanos suponen una declaración universal de valores para la convivencia.

Que los alumnos/as disfruten de entornos educativos pacíficos, preocupa a partes iguales a las instituciones educativas (en 2007, se estableció el Observatorio Estatal de la Convivencia Escolar, R.D 275/2007, 23 de febrero, Boletín Oficial del Estado, Núm.64), familias, sociedad, y opinión pública. "Tampoco hay que pasar por alto que estamos en una sociedad con altas dosis de violencia y que la escuela no es un campo aislado" (Martínez, 2007, p.15). Muestra de esta preocupación generalizada, es la última campaña lanzada por Mediaset. "Se buscan valientes". Una campaña dirigida a la sensibilización y ayuda en situaciones de acoso, en el entorno escolar.

La mayoría de nosotros hemos oído en los medios de comunicación, o a través de otras personas, tal vez, incluso hemos vivido personalmente, agresiones, ya sean verbales, físicas o de otra índole, que unos menores infligen a otros en secundaria, pero también en los niveles iniciales de la escolaridad infantil, primaria, que suponen algún tipo de agresión al otro y que se tienden a minimizar. "Los episodios de violencia en el alumnado no brotan del vacío. En gran medida suelen ser la punta de un iceberg, que está compuesto por la compacta red de relaciones interpersonales que configuran la estructura social de la institución educativa" (Ortega, 2003, p.79),

Algunas veces, existe una tendencia a quitar importancia a estos hechos con el consabido "... cosas de críos" dejando, sin argumentos, es decir indefensos, a los agredidos y a sus familias, permitiendo la violencia en los centros educativos, y proporcionando el sustrato para el incremento de la misma, alejándonos de entornos educativos pacíficos. 
Blanchard y Muzas (2007, p.7), evidencian que, en la actualidad la educación tiene como objetivo fundamental la adecuada convivencia en los centros escolares y en las aulas.

Educar para la paz, utilizar la mediación para la resolución de los conflictos, logrando entornos educativos pacíficos, en las instituciones educativas, en los centros escolares, en las aulas de éstos centros, se configura como un aprendizaje, una práctica principal para el individuo y para la sociedad, pero también es un requisito previo para otros aprendizajes, por lo tanto, constituye en sí un aprendizaje indispensable, además de ser salvaguardia de una convivencia adecuada en el centro educativo y en la sociedad.

"El reconocimiento, en definitiva, de que todo diseño educativo ha de incluir un componente ético que debe adaptarse a las nuevas condiciones sociales y que debe dar sentido al resto de conocimientos."(MEC,1993, p.10).

"Dicho con otras palabras, el sistema educativo, como espacio de aprendizaje y convivencia, debe facilitar los instrumentos necesarios para hacer posible el aprendizaje de la cultura de paz y la no violencia..." (Jares, 2004, p.12).

Los centros educativos, están comprometidos a ser entornos pacíficos, sitios, donde se enseñen valores fundamentales, pero donde también esos valores se pongan en práctica, impregnando el día a día del centro, no limitando su aprendizaje a unas horas de la jornada escolar. Pieza esencial de la educación para la paz en las instituciones educativas, es el tratamiento adecuado de los conflictos a través de la mediación, controversias que surgen de forma natural en las diferentes situaciones de la vida del centro. Se debe dar solución a los mismos cuando aparecen como prevención al incremento de violencia que supone, que éstos no se resuelvan "...la existencia de un buen clima de relaciones sociales, disminuye el riesgo de violencia”. (Ortega, 2003, p.79).

"Se trata de favorecer la creación de un ambiente de "apoyo" de "pertenencia" donde se atiende dentro de lo posible las necesidades individuales de los miembros con una ética de preocupación mutua". (Fernández, 1998, p.15).

Los conflictos, se deben solucionar, cuando aparecen, para que no se incrementen y se hagan poderosos, tenemos el encargo de solventarlos, cuando son pequeños, prestarles especial atención para que no impidan la existencia de un adecuado clima institucional, alcanzando de esta manera entornos educativos pacíficos, que finalmente se convertirán también entornos familiares y sociales pacíficos.

"De ahí que la intervención preventiva, es decir la que busca la creación de un buen clima de convivencia, sea la mejor medida para evitar la aparición de abusos, y malos tratos de todo tipo". (Ortega, 2003, p.79).

Para Acosta (2008, p.138), el conflicto y el desacuerdo son imprescindibles para mejorar, pero es importante saber gestionar los conflictos para que se conviertan en un factor de enriquecimiento. Por ello se propone utilizar la Mediación en las instituciones educativas, para la resolución de conflictos, como herramienta de paz. 
Educar para la paz en las instituciones educativas, como hemos visto, es tener como referente los Derechos Humanos, es educar para disfrutar de entornos educativos pacíficos, pero también es gestionar los conflictos que se producen en las instituciones educativas, a través de la mediación.

Jares (2004, p.54) considera la educación para los derechos humanos como un proceso educativo continuo y permanente ligado al concepto de desarrollo, paz, democracia y perspectiva positiva del conflicto.

En este sentido, la Unión Europea promulgó la directiva 2008/52/CE Parlamento Europeo y del Consejo del 21 de mayo de 2008, que abogaba por el uso de la mediación para resolución de conflictos civiles y mercantiles entre los Países miembros. En esta directiva, se establecen las normas básicas del proceso mediación, se define la mediación, se habla de los principios, de la calidad de la misma, y también que promueve la resolución de conflictos de forma amigable, etc. No olvidemos que, entre otros, uno de los objetivos de la Unión Europea es mantener a Europa unida y en paz por ello propone la mediación como una herramienta esencial para la solución de controversias, de carácter transfronterizo entre los países miembros, ya que supone solucionar las diferencias de manera pacífica, lo que genera, a su vez, entornos de convivencia pacífica, y finalmente relaciones beneficiosas entre los diferentes países.

Llegamos al convencimiento de lo trascendental del uso de la mediación para resolución de las desavenencias, como herramienta de paz, que genera a su vez entornos apacibles, que permiten la convivencia posterior a la resolución del conflicto, por ello se considera también su utilización en las instituciones educativas, ya que es un instrumento para la paz entre las personas, que genera a su vez paz, y por lo tanto posibilita entornos educativos pacíficos.

\section{LA MEDIACIÓN COMO RESPUESTA A LOS CONFLICTOS}

Vamos a detenernos en la mediación, como medio, para la resolución de conflictos en las instituciones educativas. Consideraremos algunos aspectos a tener en cuenta:

La mediación, se ha definido como un proceso de resolución cooperativa del conflicto Kruk (1997). También "Es un método de resolución de conflictos en el que dos partes enfrentadas recurren voluntariamente a una tercera persona imparcial, el mediador, para llegar a un acuerdo satisfactorio" (Torrego, 2013, p.11).

Como vemos, la mediación se define como un proceso voluntario (existe una voluntad inicial de solucionar la situación por parte de todos los integrantes del proceso) en el que dos o más partes en desacuerdo reciben la ayuda de otra persona (o personas) neutral para comunicarse y alcanzar un acuerdo conveniente a todos sobre los aspectos objeto de controversia. Es muy importante la cooperación entre todos los afectados para conseguir resolver el conflicto.

La mediación es un instrumento de resolución de conflictos, ayuda a solucionar controversias, utilizar la mediación es decisivo para la ciudadanía si queremos conseguir sociedades cada vez más justas y pacíficas, mas 
evolucionadas a la hora de resolver confrontaciones. Ya que a través de la mediación todas las partes son vencedoras, instaurando un poso de cordialidad, de afabilidad, entre los implicados.

También Femenia (2005, p.12); entre otros, muestra que será necesario respetar una serie de principios, por parte del mediador: mostrar, imparcialidad e independencia, servicio, neutralidad, confidencialidad evitar conflictos de intereses o apariencia de los mismos, voluntad y libre disposición.

Otro autor habla del "mediador como informador de la neutralidad, la imparcialidad y la confidencialidad". (Bolaños, 2005, p.54).

El espacio en el que se desarrolle la mediación también hay que cuidarlo, como no podía ser de otra manera, debe favorecer la comunicación, la confidencialidad, debe ser neutral, debe posibilitar la escucha activa y la cooperación entre las partes para resolver el conflicto. "...es importante vestir de suficiente autoridad el encuadre que presida el ritual de devolver la competencia de resolver los propios conflictos a quienes están envueltos en ellos". (Hidalgo, 2005, p.169).

A continuación presentaremos de forma ilustrativa algunas de las habilidades y técnicas utilizadas en mediación, así mismo nos iniciaremos en el conocimiento del procedimiento de mediación. "La paz, por tanto, como todo arte no se nutre sólo del talento o de las buenas intenciones, sino que requiere también su aprendizaje" " (Farré , 2010,p.16).

La mediación, como veremos, comprende una serie de Técnicas y Habilidades comunes a otros ámbitos de acción como el asesoramiento o la orientación, pero su aplicación es diferente Hidalgo (2005), ya que la mediación presenta una serie de rasgos que son propios.

En cuanto a las habilidades, técnicas y herramientas utilizadas en la mediación, (Levesque 1998; Bolaños, 2005; Torrego 2013), se identifican y presentan entre otras las siguientes: mostrar interés, técnicas de comunicación, clarificar el problema, tener una actitud creativa ante el conflicto, técnicas de negociación, etc.

Tabla 1. (Bolaños, 2005, p 48)

\begin{tabular}{|l|}
\hline - Técnicas de autoconocimiento. Creación de la propia credibilidad. \\
\hline -Técnicas de reunión. \\
\hline -Creación de confianza y relación. \\
\hline -Identificación y análisis de conflictos. \\
\hline -Valoración de intereses y necesidades de todas las partes implicadas. \\
\hline -Empatía. Capacidad de escucha. \\
\hline -Fomentar la responsabilidad de las partes. \\
\hline -Parafrasear. Reencuadre. \\
\hline -Compartir información. \\
\hline -Manejar la neutralidad. Ruptura de Patrones y Estereotipos. \\
\hline -Utilización adecuada del humor. \\
\hline
\end{tabular}




\begin{tabular}{|l|}
\hline -Fijar metas y objetivos. \\
\hline -Planificar estrategias para conseguir objetivos. \\
\hline -Identificar y ordenar los puntos para tratar. \\
\hline -Tener una actitud creativa del conflicto \\
\hline -Técnicas de negociación. \\
\hline -Técnicas de Comunicación. \\
\hline -Redacción de acuerdos. \\
\hline
\end{tabular}

También, Armas (2003), nos muestra la clasificación que realiza Levesque (1998), sobre las estrategias y técnicas de mediación.

Tabla 2. Levesque (1998)
Escucha activa
Reflejar
Recogida de información
Aporte de información
Utilización de un lenguaje neutro
Decidir el ritmo de la entrevista
Utilizar el humor
Parafrasear y Resumir
Individualizar y reformular
Universalizar y confrontar
Reencuadrar e interpretar
Previsión de dificultades
Acento sobre similitudes
Favorecer la toma de decisiones.

Vemos que la información presentada en ambos cuadros, nos proporciona una visión sobre las técnicas y habilidades que debe tener presente el mediador para llevar a cabo el proceso de mediación...

Torrego JC, (2013, p.78), en su libro sobre la mediación de conflictos en instituciones educativas también muestra las Técnicas de la escucha activa.

Tabla 3. Torrego, (2013, p.78). Técnicas de la escucha activa.

\begin{tabular}{|l|l|l|l}
\hline Habilidad/Técnica & Objetivos & Procedimiento & Ejemplos
\end{tabular}




\begin{tabular}{|c|c|c|c|}
\hline Mostrar interés & $\begin{array}{l}\text { Interesarnos por } \\
\text { lo que nos dice el } \\
\text { otro. } \\
\text { Fomento que el } \\
\text { interlocutor nos } \\
\text { cuente cosas. }\end{array}$ & $\begin{array}{l}\text { "Utilizar palabras } \\
\text { neutrales" }\end{array}$ & $\begin{array}{l}\text { "¿Puedes } \\
\text { contarme más } \\
\text { sobre este } \\
\text { asunto? }\end{array}$ \\
\hline Clarificar & $\begin{array}{l}\text { Aclarar lo dicho, } \\
\text { se utiliza para } \\
\text { obtener más } \\
\text { información. } \\
\text { Observamos otros } \\
\text { puntos de vista. }\end{array}$ & $\begin{array}{l}\text { Preguntar. Pedir } \\
\text { que se aclare algo } \\
\text { que no se ha } \\
\text { entendido. }\end{array}$ & $\begin{array}{l}\text { ¿Desde cuándo } \\
\text { ocurre esto? ¿Y } \\
\text { tú entonces, que } \\
\text { hiciste? }\end{array}$ \\
\hline Parafrasear & $\begin{array}{l}\text { Demostrar que } \\
\text { estamos } \\
\text { comprendiendo lo } \\
\text { que pasa. } \\
\text { Verificar el } \\
\text { significado. }\end{array}$ & $\begin{array}{l}\text { Repetir ideas y } \\
\text { asuntos básicos e } \\
\text { importantes. }\end{array}$ & $\begin{array}{l}\text { "Entonces tú } \\
\text { dices que..." } \\
\text { "Si no he } \\
\text { entendido mal lo } \\
\text { que tu comentas } \\
\text { es...? }\end{array}$ \\
\hline Reflejar & $\begin{array}{l}\text { Mostrar que se } \\
\text { entienden los } \\
\text { sentimientos del } \\
\text { que habla. }\end{array}$ & $\begin{array}{l}\text { Mostrar los } \\
\text { sentimientos del } \\
\text { interlocutor. }\end{array}$ & $\begin{array}{l}\text { "Te duele este } \\
\text { asunto..." } \\
\text { "Te preocupa esta } \\
\text { situación". }\end{array}$ \\
\hline
\end{tabular}

Prestar especial atención a la escucha activa, la forma de obtener información, a cómo esta se produce y la forma de comunicar por parte del mediador será también substancial en el proceso. "Recordemos que los instrumentos de intervención de resolución de conflictos como la negociación o la mediación, son esencialmente comunicativos. Se basan en relaciones comunicativas entre personas, facilitadas, en su caso, por un tercero imparcial" (Farré, 2010 ,p. 55).

En el proceso de mediación, conviene sobre todo centrarse en el presente y mirar hacia el futuro como un estado ideal libre de controversia, en el que estemos felices tranquilos y relajados, pero para llegar a ese estado entre todos tenemos que dar solución al enfrentamiento.

"Los mediadores tienden a centrarse desde el principio en el presente y el fututo, sin preguntas sobre el pasado". (Parkinson, 2005, p.118). Intentar visualizar el futuro, ayuda a la solución del conflicto.

"Las preguntas orientadas hacia el porvenir facilitan que dejen atrás las ofensas, y heridas y que piensen en cómo desean que sea el mañana." (Parkinson, 2005, p. 118).

Conseguir la cooperación entre las partes es conseguir mediar con éxito. Moore (1986), identifica algunos aspectos que obstaculizan la cooperación, por 
ejemplo: las emociones desmedidas, los estereotipos, los prejuicios, ausencia de legitimidad y/o confianza y finalmente baja comunicación.

Una vez iniciado el trabajo conjunto con las partes en conflicto, será pertinente considerar algunos aspectos. Resulta interesante las aportación realizada por Moore (1995, p. 68), en lo referente al proceso con las partes en conflicto. Para Moore (1995, p. 68), existen una serie de etapas del movimiento del mediador y situaciones críticas que deben ser afrontadas.

Tabla 4. Moore (1995, p.68),

\begin{tabular}{l} 
TRABAJO CONJUNTO CON LAS PARTES. \\
En las sesiones de mediación: iniciar la negociación entre las partes, \\
establecer un tono positivo, delimitar temas y cuestiones para discutir, ayudar a \\
las partes a explorar los compromisos, los aspectos destacados y la influencia. \\
\hline $\begin{array}{l}\text { Definición de las cuestiones y elaboración de una agenda: identificar } \\
\text { temas de interés, obtención de acuerdos, secuenciar el tratamiento de temas. }\end{array}$ \\
Revelación de intereses ocultos de las partes: identificar los aspectos \\
que son importantes para cada una de las partes en conflicto y la actitud ante \\
los mismos, ya sean intereses económicos, sentimentales, de procedimiento, \\
etc. \\
importancia de generar alternativas, generar opciones que utilicen la \\
negociación basándose en intereses. \\
Evaluación de las alternativas: revisión de intereses de las partes, en \\
que mediada hay alternativas para satisfacer dichos intereses, valoración de \\
los costes y beneficios de las diferentes alternativas. \\
\hline $\begin{array}{l}\text { Negociación definitiva: alcanzar acuerdos a través de la cooperación, } \\
\text { aproximando posiciones. }\end{array}$ \\
Acuerdo formal.
\end{tabular}

Este cuadro nos ayuda a clarificar los aspectos que debemos tener presentes durante la mediación.

Para Farré (2010), la mediación comprende una serie de etapas, pero también hace referencia a la fase previa a la mediación, "Las fases previas a la mediación propiamente dicha constituyen lo que John Paul Lederach denomina la entrada en el proceso de mediación" (Farré, 2010, p.129). En esta etapa previa a la mediación, entre otros aspectos el mediador reflexiona sobre su función, sobre las técnicas y herramientas a utilizar en la mediación, sobre la naturaleza del conflicto, etc...

Tabla 5. Farré $(2010, .129)$. Las fases de la mediación.

-La pre-mediación: preparación y consideración del conflicto. 


-La entrevista conjunta de información.
-Las entrevistas individuales.
-Preparación para la sesión de mediación.
-Inicio de la sesión de mediación.
-Cierre de la sesión o final de la mediación.
-Los resultados de la mediación.

También Torrego (2013, pp.97-98), nos ofrece una serie de pasos para acometer el proceso de mediación, incluye una fase de premediación. Siguiendo a este autor y su conocimiento sobre la mediación en conflictos en instituciones educativas, se ha elaborado el siguiente cuadro.

Tabla 6. Torrego (2013, pp.97-98).

\begin{tabular}{|c|c|}
\hline Premediación & $\begin{array}{c}\text { Información sobre la } \\
\text { mediación y acceso a la } \\
\text { misma. }\end{array}$ \\
-Presentación de las partes en \\
conflicto e información sobre aspectos \\
importantes de la mediación. \\
-Cada parte comunica su versión del \\
problema origen de la controversia. \\
-Dar objetividad al problema. \\
-Generar opciones entre todos, para \\
resolver el problema. Comprobar de forma \\
cooperativa la viabilidad de las opciones \\
propuestas. Elegir la opción, adecuada entre \\
todos. \\
-Fase de acuerdo. Redactar el \\
acuerdo de forma clara, detallada, y con \\
asunción de compromisos concretos, por las \\
partes que acuden a la mediación.
\end{tabular}

Como hemos visto anteriormente, para algunos autores, la fase previa a la mediación es fundamental antes de comenzar con la misma.

En este documento se ha tratado de acercar y dar a conocer de forma concisa, lo que es la mediación y lo que comporta el proceso de mediación. En el artículo se han presentado, los beneficios de la mediación, los principios, las fases, los pasos, del proceso de mediación, a través de las aportaciones de diferentes autores como Moore (1995); Farré (2010) y Torrego (2013). Así como las principales habilidades, técnicas y herramientas, utilizadas para 
acometer con éxito la mediación siguiendo a Armas (2003), Bolaños (2005) y Torrego (2013), entre otros autores.

A continuación, se muestra una experiencia muy interesante en la que se la pone en práctica la mediación con éxito, en una Institución Educativa. Vemos la práctica de la mediación en situaciones reales, en los centros educativos, en esta caso, a través profesores/as ayudantes y/o de alumnos/as ayudantes, con formación en mediación. Basadas en el asesoramiento, coordinación y respaldo de los primeros a los segundos, ayudando todos para mediar y solucionar conflictos en el propio centro educativo, para resolver situaciones inicialmente conflictivas, que suponen una práctica idónea para conseguir entornos educativos pacíficos, por la cercanía y la prontitud con la que se resuelven los conflictos, evitando que se perpetúen e incrementen.

La Mediación en la institución educativa, por lo tanto, puede llevase a cabo tanto por profesores/as, como por alumnos/as, formados en mediación, aprendiendo y enseñando a convivir para disfrutar de entornos educativos pacíficos, libres de violencia, integradores.

A continuación, se presenta como referente y modelo, la experiencia del Instituto público de Enseñanza. Secundaria, José Saramago, situado en Arganda del Rey (Madrid). Centro pionero en el municipio de Arganda del Rey, en la utilización de la mediación para la solución de conflictos a través de profesores/as ayudantes y alumnos/as ayudantes que asumen de forma altruista, la función de mediadores, en las situaciones conflictivas que surgen en el día a día del centro educativo, para garantizar una óptima convivencia en el mismo.

Se configura como modelo de convivencia en el entorno educativo, que se encuentra, a su vez, dentro de un programa solidario que se desarrolla en el centro.

Resulta un modelo, válido útil, orientativo, aplicable, a otras realidades educativas (adaptándolo a las características de cada centro), que da respuesta a las demandas, de la sociedad, de las familias y de los centros educativos, para conseguir algo tan esencial y necesario, para el individuo y para la sociedad en la que éste vive, como es la consecución de entornos educativos pacíficos, libres de violencia en cualquiera de sus manifestaciones.

Esteban (2013, p.175), nos habla de la pérdida de valores en la sociedad actual, de la importancia de reforzar la convivencia en los centros educativos. Una forma de hacerlo es a través de los alumnos/as como protagonistas de la creación de ambientes donde la convivencia sea una realidad.

Este modelo de convivencia pacífica, utilizando la mediación para la solución de conflictos es organizado y llevado a la práctica por el director del centro D. José Fernández Medina, la coordinadora de alumnos/as ayudantes y profesores/as ayudantes $D^{\underline{a}}$ Sagrario Pulido Varo así, como por el equipo de profesores/as ayudantes y alumnos/as ayudantes que ejercen la mediación para la solución de conflictos. Siempre fuera del horario de clases. Entre todos contribuyen a la óptima convivencia en este instituto de enseñanza secundaria de Arganda del Rey, con una trayectoria de mediación en conflictos, muy satisfactoria. 
Paralelo, a la práctica de la mediación para la resolución de conflictos, en este centro, está el régimen disciplinario del mismo, el director del centro nos dice que, la mediación ni conmuta, ni enmascara. Pero, si es el caso, una vez aplicada la sanción, la mediación tiene la bondad de restaurar la convivencia entre los implicados en el conflicto reduciendo o eliminando las probabilidades de que el hecho conflictivo se produzca de nuevo.

Existe un grupo de profesores/as que voluntariamente, y con una formación previa en mediación ejercen como profesores/as ayudantes utilizando la mediación en situaciones conflictivas, en el centro, son 4 o 5 profesores. A su vez existe un grupo de alumnos/as, suelen ser dos o tres por cada clase que se ofrecen como voluntarios, para ser alumnos/as ayudantes entre otros aspectos median en conflictos, ayudan a integrar compañeros, nuevos o aislados, etc. Estos alumnos/as que comienzan siendo ayudantes en los primeros niveles ( $1^{\circ}$ y $2^{\circ}$ de la E.S.O), al final de la secundaria obligatoria y/o de bachillerato gracias a su experiencia como ayudantes en los niveles iniciales y sus conocimientos se convierten en alumnos/as ayudantes que median también, cuando se requiere una mediación de carácter más formal. Siempre contando estos alumnos/as ayudantes que median en conflictos, con formación, apoyo, asesoramiento, observación y ayuda, de los profesores/as ayudantes del centro, de la coordinadora y en última instancia del Director. Todos unidos para garantizar la adecuada convivencia en el centro educativo. Están en contacto unos con otros, coordinados. Siempre fuera del horario de clases. Habitualmente en el recreo.

La mayoría de las situaciones conflictivas que brotan en el centro, se resuelven de esta manera, es decir a través de los alumnos/as ayudantes coordinados por los profesores/as ayudantes. Son conflictos iniciales, "cogidos a tiempo", por lo tanto con un pronóstico favorable para su solución, a través de la mediación. En ocasiones, se pasa a un segundo nivel, en el que la mediación adquiere un carácter más formal, ya sea con alumnado ayudante experimentado ejerciendo como mediadores $\mathrm{y} / \mathrm{o}$ de profesores ayudantes ejerciendo de mediadores, o la propia coordinadora del alumnado y el profesorado ayudante.

Los alumno/as ayudantes, reciben una formación previa en mediación en instalaciones del municipio. En las "Jornadas de formación de alumnos/as ayudantes". Que suelen realizarse en sábado o por la tarde, fuera del horario de clases.

Es importante dedicar especial atención a los chicos que llegan cada año nuevos al centro, los alumnos/as de primero de la E.S.O Determinar los ayudantes y formarles. En ocasiones desde el centro se pregunta a los profesores/as de 6을 de primaria de diferentes colegios, que alumnos/a desempeñarían adecuadamente la función de alumnos/as ayudantes en el I.E.S.

Desde la coordinación, se organiza, que determinados profesores/as ayudantes, asesoren y organicen a su vez, a determinados alumnos/as ayudantes por cursos, por ejemplo: un profesor/a ayudante se ocupa de los alumnos/as ayudantes de primero de E..S.O, otro de los de segundo y así sucesivamente. 
Los tutores/as (a veces coincide que los tutores son también profesorado/ayudante) tienen una labor muy importante, motivan, animan, sensibilizan el proceso. A través de información sobre la solidaridad en el centro educativo, se destaca la importancia de ayudar a los demás, de convivir en un entorno pacífico.

Una vez elegidos los alumnos/as ayudantes, reciben la formación para ejercer como mediadores en los conflictos. Estos alumnos/as son conocidos por la clase, respetados, la clase los percibe como un apoyo, pues ayudan a los compañeros, las familias perciben como algo bueno que su hijo/a sea ayudante, y también que existan alumnos/as ayudantes en el centro para garantizar una buena convivencia en el mismo.

Los pasos que siguen, habitualmente, los alumnos/as ayudantes cuando median en los conflictos iniciales entre compañeros, son entre otros, los siguientes:

Existe una primera fase, muy importante de premediación, en la que se informa con claridad que los alumnos/as ayudantes son personas que amplían su campo de visión con el único fin solidario de ayudar a los demás, de ayudar a sus compañeros. En ningún caso son "chivatos, ni "pelotas del profesor". No son ayudantes del profesor, son ayudantes de otros compañeros. Actúan ante las necesidades del otro.

Cuando surge un conflicto, los alumnos/as en conflicto, o uno de los alumnos/as en conflicto, a veces también puede ser un tercero otro compañero, o un amigo... etc, manifiesta lo ocurrido al alumno/a ayudante 0 al profesor/a ayudante, o al tutor/a del curso, o a la coordinadora.

Los alumnos/as ayudantes, son activos y están atentos a los que les rodea.

Si ellos ven lo sucedido, o se enteran de lo ocurrido, pueden resolver ese conflicto inicial, en clase o en el recreo, con los implicados. Comienzan clarificando el conflicto. Intentando llegar a una solución conjunta del conflicto y a un acuerdo, que incluye respeto al otro, o entre las dos partes en conflicto, dependiendo de la situación. Después se informa de lo ocurrido al profesor ayudante, y/o a la coordinadora.

En otras ocasiones, si no han presenciado el momento conflictivo, intentan averiguar lo ocurrido con los implicados, investigan e informan al profesor ayudante. Si el alumno ayudante, no puede resolverlo, intervendría, el profesor/a ayudante y/o la coordinadora. En función de cada caso.

La mayoría de los conflictos se resuelven de esta manera, desaparecen, pues utilizan la mediación, nada más producirse el hecho conflictivo. Evitando que este se haga grande, poderoso.

Hay otras situaciones, las menos, en las que el conflicto, continua, en estos casos es necesario realizar una mediación más formal. Se comienza con una primera fase de premediación, se informa a las partes de los principios de la mediación, imparcialidad, neutralidad, voluntariedad, etc. Se resalta la importancia de la comunicación para resolver situaciones, se destacan los beneficios de la mediación con ella, no se pierde nada, al contrario, si se resuelve la situación todos salen ganando. 
Se busca un horario y se determina un tiempo y un espacio para la mediación, (siempre fuera del horario de clases), durante el recreo, alguna hora libre, etc.

Se conversa con los implicados, y se les cuenta en qué consiste la mediación, Se les explica que es un proceso independiente de la medida disciplinaria. Esto es muy importante resaltarlo, si hubiese medida disciplinaria no se detiene, de esta forma, el alumnado que comete faltas de disciplina no se ampara en la mediación para evitar la sanción.

Si las partes están conformes con el procedimiento de mediación, (no olvidemos que la mediación es un proceso voluntario) y también con los alumnos/as ayudantes (experimentados)y/o en su caso con los profesores/as ayudantes que actuarán como mediadores para la solución del conflicto(para evitar conflicto de intereses), se comienza el proceso de mediación, que entre otras tiene las siguientes fases:

Tabla 7. Fases del proceso de mediación en el I.E.S José Saramago.

1 Cada parte informa del conflicto.

2. Se identifican intereses, de ambas partes.

3. Entre todos tratan de definir el conflicto objetivamente.

4. Se ponen en el lugar del otro.

5. Negociación cooperativa.

6. Acuerdo, por escrito.

7. Revisión del acuerdo semanal y quincenal.

Hemos presentado este modelo que comporta la aplicación práctica de la mediación en un centro educativo consiguiendo entornos educativos pacíficos. Es una realidad, es posible, y también una prioridad, en el momento actual en los centros educativos.

Pero para que se extienda a más centros, para dar respuesta a esta necesidad educativa y social, es decisivo que en los horarios lectivos de estos centros se disponga de un tiempo específico, para la formación y la práctica de la mediación.

La práctica de la mediación en los centros educativos para la resolución de conflictos es algo tan importante, es algo tan esencial, que supone, que los alumnos/as disfruten de entornos educativos pacíficos, de formación, de crecimiento en su diferentes espacios, entornos integradores, no excluyentes, a los que los alumnos/as quieran asistir para aprender, en los que haya un buen clima institucional, y a los que ningún alumno/a con ganas y derecho a la educación, deje de asistir, por miedo a algún tipo de violencia en sus diferentes manifestaciones, por situaciones conflictivas iniciales no resueltas, que se han ido incrementando, derivando en acoso escolar.

Se ha querido resaltar, como no podía ser de otra forma, la repercusión de la educación en valores para el individuo y para la sociedad en la que éste vive. Una Educación en valores que debe ser ofrecida preferentemente desde el ámbito familiar y posteriormente desde el ámbito educativo, hallándose 
después escuela y familia acompasadas en esta transmisión, ya que supone un aprendizaje imprescindible para la persona, que beneficia a todos.

Comprendida en esa educación en valores, se tiene certeza de la trascendencia de educar para la paz, utilizando la mediación en las instituciones educativas como herramienta preventiva y de resolución de conflictos, a fin de conseguir entornos educativos pacíficos, como factor de calidad y mejora de la educación en las instituciones educativas.

El presente y el futuro de los individuos, de la sociedad en su conjunto y del progreso de ambos, pues su avance es reciproco, se encuentra en gran medida, en la conquista de entornos educativos pacíficos, valiéndonos de la mediación para la resolución de conflictos, en instituciones educativas. Gracias a ella contemplaremos los centros educativos como escenarios pacíficos, libres de violencia, como medios esencialmente formativos, de respeto a la dignidad individual, de enriquecimiento personal y social, en todos y cada uno de sus emplazamientos.

\section{REFERENCIAS BIBLIOGRÁFICAS}

Aclann, A.F. (1990). Cómo utilizar la mediación para resolver conflictos en las organizaciones. Barcelona: Paidós.

Acosta, A. (2008). Educación emocional y convivencia en el aula. Madrid: Egraf.

Aieef, S. (1985). Análisis sociológico de la familia española. Barcelona: Ariel.

Alzate, R. (1998). Análisis y resolución de conflictos. Una perspectiva psicológica. Bilbao: Servicio Editorial Universidad del País Vasco.

Armas, M. (2003). La mediación en la resolución de conflictos. La gestión del conocimiento, 32, pp125-136.

Barona, S. (1999). Solución extrajudicial de conflictos. Alternative dispute resolution $(A D R)$ y Derecho Procesal. Valencia: Tirant lo Blanch.

Blanchard, M. \& Muzás D. (2008). Acoso Escolar/School Harassment: Desarrollo, prevención y herramientas de intervención. Madrid: Narcea.

Biffi, S. (2010). Camino de encuentro y descubrimientos: dinámicas y vivencias. Bogotá: San Pablo.

Bernal, T. (2002). La mediación. Una solución a los conflictos de pareja. Madrid: Colex.

Bolaños, I. (2005). El mediador familiar. Madrid: Uned.

Bonals, J \& Sánchez, M. (2007). Manual de asesoramiento psicopedagógico. Barcelona: Grao.

Boqué, C. (2003). Cultura de Mediación y Cambio social. Barcelona: Gedisa.

Bowlby, J. (2006). Vínculos afectivos. Formación desarrollo y pérdida. Madrid: Morata. 
Bustelo, D. (1993). La mediación interdisciplinaria familiar. Madrid: Alfaomega.

Cárdenas, E. J. (1999). La mediación en los conflictos familiares. Buenos Aires: Lumen Humanitas.

Chorda, R. (1991). ¿Negociaciones? Madrid: Rama.

Díez, F. \& TAPIA, G. (1999). Herramientas para trabajar en mediación. Buenos Aires: Paidós.

Doltó, F. (1986). La causa de los niños. Buenos Aires: Paidós.

Doltó, F. (1989). Cuando los padres se separan. Buenos Aires: Paidós.

Eglulz, L. (2003). Dinámica de la familia: un enfoque psicológico y sistémico. México: Pax.

Ekman, P. (1985). Cómo detectar mentiras. Barcelona: Paidós.

Elosegui, M. (2002). Diez temas de género. Madrid: Ediciones Internacionales Universitarias.

Espina, A. (2004). Familia, educación y diversidad cultural. Salamanca: Universidad de Salamanca.

Esteban Moreno, R.M. (2003). Educación en valores. Programa para su desarrollo en la Educación Secundaria Obligatoria. Tendencias pedagógicas, vol. (8), pp.99-108.

Esteban Moreno, R.M. (2013). Los iguales, protagonistas en la resolución de conflictos y en la mejora de la convivencia. Actas del III Congreso Internacional de Convivencia escolar. Granada: Geu.

Farré, S. (2010). Gestión de conflictos: taller de mediación. Barcelona: Planeta.

Femenia N. (2006). Una teoría posmoderna de conflictos sociales. Florida International University. Transformación de conflictos en el mundo hispano [Internet] 2005. Disponible en: http://www.intermediacion.com/conflictos_sociales.htm

Fernández, I. \& Torrego, J.C.(2001). La mediación en la escuela. En actas del curso de verano de la UNED: Familias: Respuestas educativas y sociales. Madrid Ministerio de Trabajo y Asuntos Sociales.

Fernández, I. (1998). Prevención de la violencia y resolución de conflictos. Madrid: Narcea.

Ferre, S. (2004). Gestión de conflictos: taller de mediación. Barcelona: Ariel.

Fisher, R. \& Ertel, D. (2000). Obtenga el sí en la práctica. Cómo negociar paso a paso ante cualquier situación. Barcelona: Gestión 2000.

Fisher, R. \& Ury, W. (1984). Obtenga el sí: el arte de negociar sin ceder. Proyecto de investigación sobre negociación de Harvard. México: Compañía Editorial Continental.

García, L. (2003). Mediación familiar. Prevención y alternativa al litigio en los conflictos familiares. Madrid: Dykinson. 
García, M. (2004). Programa de acción tutorial para la educación secundaria obligatoria. Barcelona: Praxis.

García, L. (2006): Mediación en conflictos familiares: una construcción desde el derecho de familia. Madrid: Ed. Reus.

García, L. (2007). Mediación en sede de adopción en Los menores en protección. Madrid: Ed. Difusión Jurídica y Temas de actualidad. S.A.

Gimeno, A. (1999). La familia: el desafío de la diversidad. Barcelona: Ariel.

Gómez-Pomar, J. (1991). Teoría y Técnicas de Negociación. Barcelona: Ariel.

Haley, J. (1985). Terapia para resolver problemas. Buenos Aires: Amorrortu.

Haynes, J.M. (1995). Fundamentos de la mediación familiar. Madrid: Gaia.

Herscovici, P.(1991). El divorcio y las nuevas organizaciones familiares. Buenos Aires: Nadir.

Hicks, D.(1993). Educación para la paz. Cuestiones, principios, y práctica en el aula. Madrid: Morata.

Hidalgo, F. (2004). Curso de mediación y orientación familiar vol.Il. Madrid: UNED.

Hoffman, L. (1984). Principi di terapia della familia. Roma: Astrolabio.

Jares, X. (1999). Educación para la paz. Su teoría y su práctica. Madrid: Popular.

Jares, X. (2001). Educación y conflicto. Guía de educación para la convivencia. Madrid: Popular.

Jares, X. (2004). Educar para la paz en tiempos difíciles. Bilbao: Bakeaz.

Jiménez, A. (2005). Modelos y realidades de la familia actual. Madrid: Fundamentos.

Judson, S.(1985). Aprendiendo a resolver los conflictos. Manual de educación para la paz y la no violencia. Barcelona: Lerna.

Keeney, E. (1985). L'estetica del cambiamiento. Roma: Astrolabio.

Kurt, L, (1996). Terapia sistémica. Barcelona: Herder.

Lansberg, I. (2000). Los sucesores en la Empresa Familiar. Barcelona: Granica.

Lebel, P.(1990). El arte de la negociación. Barcelona: Ceac.

Lederach, J.P. (1984). Educar para la Paz. Objetivo Escolar. Barcelona: Fontamara.

Lederach, J.P. (2000). El abecé de la paz y los conflictos. Educación para la paz. Madrid: Los libros de la catarata.

Le Poole, S. (1989). Nunca aceptes un no por respuesta. Bilbao: Deusto.

Ley orgánica 8/1985, de 3 de julio, Reguladora del Derecho a la Educación. Boletín Oficial del Estado, núm. 159, 14 julio de 1985:21016. 
Ley orgánica 1/1990 de 3 de octubre de Ordenación General del Sistema Educativo Boletín Oficial del Estado, núm.238, 4 octubre de 1990:2893 y 28931.

Ley orgánica 9/1995, de la Participación, la Evaluación y el Gobierno de los centros docentes Boletín Oficial del Estado, núm.278, 20 de noviembre de 1995,33654.

Ley orgánica 10/2002 de 23 de diciembre de Calidad de la Educación.

Boletín oficial del Estado, núm.307, 24 de Diciembre de 45192.

Ley orgánica 2/2006, de 3 de mayo, de Educación. Boletín Oficial del Estado, Núm.106, 4 de mayo de 2006:17158.

Ley orgánica 8/2013, de 9 de diciembre, para la Mejora de la Calidad Educativa. Boletín Oficial del Estado, (Núm.52, 1 de marzo de 2014:19356.

Levesque, J. (1998). Méthodologie de la médiation familiale. Canadá: edisem, inc.

López, R. \& Marín, J.J. (2003). Legislación sobre mediación familia. Madrid: Tecnos.

Lucini, F. (1993). Temas transversales y educación en valores. Madrid: Alaude.

Marlon, L. (1999). Mediación familiar: Una práctica en busca de una teoría. Barcelona: Granica.

Martínez, V. (2007). La buena educación: Reflexiones y propuestas de psicopedagogía humanista. Barcelona: Anthropos Editorial.

Maslow, A. (1954). Motivation and Personality. New York: Harper.

Maturana, H. (1990). Emociones y Lenguaje en Educación y Política. Chile: Hachette.

Martínez, V. (2001). Filosofía para hacer las paces. Barcelona: Icara.

Maubert, J. (1993). Negociar: las claves del triunfo. Barcelona: Marcombo Boixareu Editores.

MEC (1993). Temas transversales y desarrollo curricular. Madrid: Mec.

Moore, C.W. (1986). El proceso de mediación. Barcelona: Granica.

Moore, CH. (1995). El proceso de mediación. Granica: Buenos Aires.

Nussbaum, M. (2010). Sin fines de lucro. España:Katz.

Ortega, P. \& Mínguez, R. (2001). Los valores en la Educación. Barcelona: Ariel.

Ortega, R. (2003). La convivencia Escolar: qué es y cómo abordarla. Sevilla: Junta de Andalucía.

Prada, J. \& López, J.A. (2007). Proyecto Armonia: mejorar la convivencia escolar. Castilla y León: Consejería de Educación de la Junta de castilla y León.

Parkinson, L. (1997). Familiy Mediation. Londres: Sweet and Maxwell. 
R.D. 275/2007, 23 de febrero, Boletín Oficial del Estado, Núm.64, 15 de marzo de 2007:11007.

Redorta, J. (1999). Herramientas en análisis de conflictos. I Simposio sobre pacificación y resolución de conflictos. Barcelona: Pau i Treva.

Rubin, J.Z., Pruit, D. G, \& Hee, K. (1994). Social Conflict: escalation, stalemate and settlement. New York: McGraw-Hill.

Saner, R. (2003). El experto negociador. Barcelona: Ediciones Gestión 2000.

Serrano, G. y Mirón, L. (2003): Problemas y perspectivas de la mediación. Actas del congreso de Psicología social. Málaga: Colegio oficial de psicólogos.

Quintana, J. M. (1983). Educación familiar en varios, Diccionario de las ciencias de la educación. Madrid: Santillana.

- (1988). Teoría de la Educación. Concepción antinómica de la educación. Madrid: Dykinson.

- (1993). Filosofía de la educación familiar en Quintana (Coord.), Pedagogía familiar. Madrid: Narcea.

Singer, L. (1996). Resolución de conflictos. Técnicas de actuación en los ámbitos empresarial, familiar y legal. Barcelona: Paidós.

Six, J.F.(1997). Dinámica de la mediación. Barcelona: Paidós.

Suares, M. (1996). Conducción de disputas, comunicación y técnicas. Barcelona: Paidós.

Torrego, J.C. (Coord). (2013). Mediación de conflictos en instituciones educativas. Manual para la formación de mediadores. Madrid: Narcea.

Truvilla, J. (2004).Convivencia escolar y resolución pacífica de conflictos. Sevilla: Junta de Andalucía.

Ury, W. (1993). De la negociación al acuerdo: claves para superar cualquier negociación. Barcelona: Parramón.

Vosler, N. (1996). New approaches to family practice: confronting economic stress. London: Sage. DOI https://doi.org/10.4135/9781483327747

Zannata, A. (1997). Le nuove famiglie. Bolonia: Mulino. 
